\title{
MicroRNA-500a Promotes the Progression of Hepatocellular Carcinoma by Post- Transcriptionally Targeting BID
}

\author{
Leilei Bao ${ }^{\mathrm{a}, \mathrm{c}}$ Mingjian Zhang ${ }^{\mathrm{a}}$ Shu Han ${ }^{\mathrm{b}}$ Yangyang Zhan ${ }^{\mathrm{a}, \mathrm{d}}$ Wenyuan Guo ${ }^{\mathrm{b}}$ \\ Fei Teng ${ }^{b}$ Fang Liu ${ }^{b}$ Meng Guo a,b Luding Zhang ${ }^{b}$ Guoshan Ding ${ }^{b}$ \\ Quanxing Wang ${ }^{\text {a }}$
}

aNational Key Laboratory of Medical Immunology, Second Military Medical University, Shanghai, ${ }^{b}$ Department of Liver Surgery and Organ Transplantation, Changzheng Hospital, Second Military Medical University, Shanghai, 'Department of Pharmacy, No. 411 Hospital, Second Military Medical University, Shanghai, 'State Key Laboratory of Oncogenes and Related Genes, Shanghai Cancer Institute, Renji Hospital, Shanghai Jiao Tong University School of Medicine, Shanghai, China

\section{Key Words}

Mir-500a • Progression • Prognosis • BH3-interacting death agonist • Hepatocellular carcinoma

\begin{abstract}
Background/Aims: Hepatocellular carcinoma (HCC) is one of the most common human malignant diseases in the world, and the mechanisms underlying HCC carcinogenesis and progression need further investigation. MicroRNAs play important roles in the development of cancer, and miR-500a is suggested to be deregulated in some types of cancer. However, the underlying molecular mechanisms of miR-500a in HCC remain unknown. Methods: The expression of miR-500a in HCC was analyzed in The Cancer Genome Atlas (TCGA) database and examined in 33 pairs of HCC tissues and matched nontumor tissues. The correlation between miR-500a expression and prognosis of HCC patients was analyzed from the survival data in TCGA. The mechanism of miR-500a upregulation in HCC was detected using chromatin immunoprecipitation-quantitative real-time PCR. The roles of miR-500a in HCC development were examined using a cell counting kit- 8 assay in vitro and growth of transplanted tumors in nude mice in vivo. Apoptosis of HCC was detected using Annexin V/propidium iodide staining. The expression of BH3-interacting death agonist (BID) protein was examined using western blot analysis. Results: miR-500a expression was upregulated in HCC tissues, and high miR500 a expression was significantly correlated with the poor prognosis of HCC patients. Histone modifications in the promoter region of miR-500a may be responsible for its increased expression. Inhibition of miR-500a in HCC cell lines significantly promoted apoptosis, as well as inhibiting the proliferation of HCC cells and growth of transplanted tumors in nude mice. miR-500a directly targeted the 3' untranslated region of BID mRNA, and inhibition of miR500 a-promoted apoptosis was almost completely abolished by the administration of ABT-199 via the BID-mitochondria pathway. Conclusion: Our results suggest that histone modifications
\end{abstract}

L. Bao, M. Zhang, S. Han, and Y. Zhan contributed equally to this work.

Quanxiang Wang

and Luding Zhang
National Key Laboratory of Medical Immunology, Second Military Medical University

Shanghai 200433, (China)

Tel. +86-21-81871006, E-Mail wangqx@immunol.org, zhangld2010@163.com 
in the promoter region of miR-500a may be responsible for the increased expression of miR$500 \mathrm{a}$ in HCC, which promotes cancer progression by targeting BID, indicating that miR-500a may be a potential prognostic predictor and therapeutic target for HCC patients.

(C) 2018 The Author(s)

Published by S. Karger AG, Basel

\section{Introduction}

Hepatocellular carcinoma (HCC) is one of the leading causes of cancer-related deaths, especially in China [1]. However, because of its complicated process with multiple factors and steps, its etiological factors and pathogenesis are still not entirely clear. Therefore, the clinical treatments for liver cancer are limited, and surgery remains the most common therapeutic approach. However, low curative rates, high recurrence rates, and heterogeneity reduce the efficacy of surgery $[2,3]$. Thus, for individualized targeted therapies of HCC, further investigation of HCC pathogenesis and the discovery of potential therapeutic targets are urgently needed.

Deregulated epigenetic modifications, including DNA and histone modifications, have been suggested to be important for the development of cancer, especially for HCC carcinogenesis and progression [4]. Epigenetic modifications can transcriptionally regulate the expression of mRNA, microRNA (miRNA), long non-coding RNA, and other genes in chromosomes or gene clusters by adjusting chromatin structure without changing the DNA sequence. In recent years, an increasing number of studies have revealed the increased expression of long non-coding RNAs [5, 6] and miRNAs [7-9] in HCC. However, due to the complexity of epigenetic modifications and limitations of research methods, we have a limited understanding of the mechanisms by which epigenetic modifications are involved in HCC processes through the regulation of gene transcription. Therefore, the roles of deregulated epigenetic modifications in the development of HCC require further investigation.

miRNAs are a class of endogenous, non-coding RNAs of approximately 20-22 nucleotides in length that are widespread in eukaryotes. These molecules account for approximately $1 \%$ of the entire human genome and are expressed widely in various tissues [10]. miRNAs can directly target the $3^{\prime}$-untranslated regions (3'-UTRs) of target genes and then inhibit the translation or induce the degradation of these mRNAs. Functional studies have confirmed that miRNAs play vital roles in various important biological processes, including proliferation, differentiation, apoptosis, and migration; thus, they have important roles in the properties of cancer biology and may serve as potential therapeutic targets $[7,11,12]$. For HCC, a set of deregulated miRNAs has been shown to participate in its carcinogenesis and progression, and the miRNome study in HCC tissues suggested some important deregulated miRNAs in HCC cancer biology, such as miR-361 [8], miR-122 [13], and miR-199 [14]. However, as multiple miRNAs are deregulated in cancer cells and a single miRNA can target multiple mRNAs, identifying the deregulated miRNAs in HCC and uncovering their roles in HCC development is an ongoing process.

In our previous study, we found that the expression of miRNA miR-500a was significantly increased in HCC as compared to normal liver according to heat map analysis of RNA sequencing in The Cancer Genome Atlas (TCGA) database [14]. Also, previous studies have shown that miR-500a is of great interest in cancer therapies due to its association with various types of cancer, including gastric cancer [15] and prostate cancer [16]. Here, we found that high miR-500a expression in HCC tissues was significantly correlated with the poor prognosis of patients; thus, we focused on the roles and corresponding mechanisms of $\mathrm{miR}-500 \mathrm{a}$ in the carcinogenesis and progression of HCC.

\section{Materials and Methods}

Cell culture and transfection

Human HCC cell lines (SMMC-7721 and HepG2), a human hepatocyte cell line (QSG-7701), and the HEK293 cell line were purchased from the American Type Culture Collection (Manassas, VA, USA). SMMC- 


\section{Cellular Physiology Cell Physiol Biochem 2018;47:2046-2055 and Biochemistry Published online:July 03, $2018 \quad \begin{aligned} & \text { DO } 2018 \text { The Author(s). Published by S. Karger AG, Basel } \\ & \text { www.karger.com/cpb }\end{aligned}$}

Bao et al.: Mechanical Studys of miR-500a in Liver Cancer

7721, HepG2, QSG-7701, and HEK293 cells were cultured in RPMI-1640 (Invitrogen, Carlsbad, CA, USA) medium containing 10\% fetal bovine serum (BioWest, Nuaillé, France) under an atmosphere of $5 \% \mathrm{CO}_{2}$ at $37^{\circ} \mathrm{C}$. For transfection experiments, $2.5 \times 10^{5} \mathrm{SMMC}-7721$ or HepG2 cells were seeded in a 6-well plate and cultured overnight, and then transfected with $50 \mathrm{nM}$ miR-500a negative controls, inhibitor, or mimics (RiboBio, Guangzhou, China) using Lipofectamine 2000 (Invitrogen) for transfection according to the manufacturer's instructions.

\section{Patient samples}

A total of 33 pairs of HCC tissues and matched adjacent liver tissues were collected from surgically resected samples at the Department of Liver Surgery and Organ Transplantation, Changzheng Hospital, Second Military Medical University (Shanghai, China). No patients had a history of chemotherapy or radiotherapy before surgery. The clinical stages of HCC tumors were determined according to the tumornode-metastasis classification system of the International Union Against Cancer (7th edition). The research protocol was approved by the Ethics Committee of the Second Military Medical University, and informed written consent was obtained from each patient according to the Declaration of Helsinki and its amendments.

Quantitative real-time polymerase chain reaction

According to our previous method [13], TRIzol (Invitrogen) was used to extract total RNA from HCC cell lines. A PrimeScript RT Reagent Kit (Takara, Shiga, Japan) was used for reverse transcription of miR500a using the stem-loop reverse transcription primer. According to the manufacturer's instructions, SYBR Premix Ex Taq (Takara) was used to perform quantitative analysis with a Step0ne Real-Time PCR System (Applied Biosystems, Foster City, CA, USA). The following primers were used: miR-500a forward reverse transcription primer: 5'-CTC AAC TGG TGT CGT GGA GTC GGC AAT TCA GTT GAG TCT CAC CC-3'; quantitative PCR forward primer: 5' $5^{\prime}$-ACA CTC CAG CTG GGT AAT CCT TGC TAC CTG G-3' and reverse primer: 5'-TGG TGT CGT GGA GTC G-3'. U6 was used as an internal control. The relative expression of miR-500a was analyzed by the $2^{-\Delta \Delta C T}$ method.

\section{Chromatin immunoprecipitation assay and real-time PCR}

The cells were cross-linked and processed according to the Millipore Chromatin Immunoprecipitation (ChIP) Assay Kit (Millipore, Billerica, MA, USA) protocol. Antibodies (5 mg) to H3K4me3, H3K9me3, H3K27me3, acH3, or control IgG (all from Millipore) were used to immunoprecipitate the respective histone residues. Sonicated preimmunoprecipitated DNA $(10 \mu \mathrm{L})$ from each sample was used as an input control. ChIP DNA was analyzed by real-time PCR as described previously [13] using the following primers. Primer 1: forward: 5'-CGA TGG AGC AAT TCG ATC GGC-3'; reverse: 5'-GGA TCG AGG CAT CGG ACG GT-3'; and Primer 2: forward: 5' -CGA TGG AGC AAT TCG ATG ATC G-3'; reverse: 5'-GCA TCC GAA GGG TAC GCA TTG C-3'.

\section{Cell proliferation assay}

Cell survival experiments were performed using the Cell Counting Kit-8 (CCK-8; Dojindo, Shanghai, China) following the manufacturer's instructions. $2 \times 10^{3}$ SMMC-7721 or HepG2 cells were seeded in 96-well plates $(100 \mu \mathrm{L} /$ well), then transfected with miR-500a inhibitor or miR-NC for $24 \mathrm{~h}$. Then, the medium was replaced with $200 \mu \mathrm{L}$ fresh medium containing 10\% FBS and began CCK8 assay, each time-point had sister group wells for detection. The cells were examined using CCK-8 $(10 \mu \mathrm{L} /$ well $)$ analysis for $2 \mathrm{~h}$ at $37^{\circ} \mathrm{C}$ and then evaluated with an automatic microplate spectrophotometer at $490 \mathrm{~nm}$ to calculate the optical density value.

\section{Apoptosis analysis}

The cells were plated in 12-well plates at a density of $1.0 \times 10^{5}$ cells/well and cultured overnight. At 24 $\mathrm{h}$ after transfection with miR-500a inhibitor, collected both floating and adherent cells. 0.25\% trypsin-EDTA was used to detach adherent cells. According to the manufacturer's instructions, the cells were stained with FITC-Annexin V and propidium iodide (BD Biosciences, Franklin Lakes, NJ, USA). Flow cytometry (Accuri C6; BD Biosciences) was performed to determine the ratio of apoptosis, and the data were analyzed using the Accuri $^{\mathrm{TM}} \mathrm{C} 6$ built-in software. Annexin V-positive cells were regarded as apoptotic cells.

\section{Dual luciferase reporter assay}

A dual-luciferase reporter assay (Promega, Madison, WI, USA) was conducted according to the manufacturer's instructions. BH3-interacting death agonist (BID) 3'-UTR fragments were obtained by PCR 
and cloned into the pGL3 vector. HEK293 cells were plated on 96-well plates at a density of $5 \times 10^{3}$ cells/ well and incubated overnight, then transfected with $80 \mathrm{ng}$ luciferase reporter plasmid, $40 \mathrm{ng}$ RL-TK-Renillaluciferase plasmid, and $20 \mathrm{nM}$ miR-500a mimics for $48 \mathrm{~h}$. Firefly luciferase activity was normalized using Renilla luciferase activity to balance transfection efficacy.

\section{Western blot analysis}

A Mitochondria/Cytosol Isolation Kit (BioVision, Inc., Milpitas, CA, USA) was used to separate cytoplasmic and mitochondrial proteins according to the manufacturer's instructions. According to our previous method [14], the obtained proteins were quantified by the BCA (Thermo Fisher Scientific, Waltham, MA, USA) method. After denaturation, equal amounts of proteins $(500 \mathrm{ng} / \mu \mathrm{L}, 10 \mu \mathrm{L})$ from different samples were separated by sodium dodecyl sulfate polyacrylamide gel electrophoresis and transferred to nitrocellulose membranes (Millipore). The blots were probed with the following antibodies: BID (ab32060; Abcam, Cambridge, UK), truncated BID (tBID) (ab108293; Abcam); cytochrome C (11940; Cell Signaling Technology, Danvers, MA, USA); cleaved caspase-3 (9654; Cell Signaling Technology); cleaved caspase-9 (7237; Cell Signaling Technology); and GAPDH (5174; Cell Signaling Technology). After washing, the membranes were incubated with a secondary antibody. Chemiluminescence (4200; Tanon Science \& Technology, Shanghai, China) was used to detect the signals, and ImageJ $1.49 \mathrm{v}$ software (National Institutes of Health, Bethesda, MD, USA) was used for quantitative analysis of the signals.

\section{Xenograft HCC model}

Animal experiments were approved by the Scientific Investigation Board of the Second Military Medical University and performed in accordance with the guidelines of the National Institutes of Health Guidance for the Care and Use of Laboratory Animals. SMMC-7721 cells $\left(2.0 \times 10^{6}\right.$ cells) were inoculated hypodermically into 6-week-old male BALB/c nude mice (Sippr-BK Laboratory Animal Co., Ltd., Shanghai, China). Two weeks later, nude mice with tumor volumes of $0.5 \mathrm{~cm}^{3}$ were injected with $20 \mathrm{nM}$ antagomiR500a-3p intratumorally every 3 days (RiboBio). Two weeks later, the mice were sacrificed and the tumors were removed to be measured and photographed.

\section{Statistical analysis}

GraphPad Prism software 6.0 (GraphPad Software, La Jolla, CA, USA) was used for statistical analysis and figure preparation. Kaplan-Meier survival analysis was used to analyze the survival data of HCC patients with a log-rank test for significance. Experimental data are expressed as the mean \pm standard deviation (SD) or as percentages. Student's $t$-test was performed to compare two groups of data. Differences were deemed statistically significant when ${ }^{*} p<0.05,{ }^{* *} p<0.01$, and ${ }^{* * *} p<0.001$.

\section{Results}

miR-500a expression is significantly increased in HCC

To investigate the expression of miR-500a in HCC, 50 cases of adjacent normal liver tissues and 288 cases of HCC tissues of miRNA chip data in TCGA were analyzed, and we found that miR-500a expression was significantly increased in HCC (Fig. 1A). Moreover, we collected 33 paired HCC tissues and matched adjacent normal liver tissues, and found that 27 of the 33 HCC samples showed significantly increased miR-500a expression using quantitative real-time PCR analysis (Fig. 1B). miR-500a expression was also significantly increased in the HCC cell lines SMMC-7721 and HepG2, but not in the liver cell line QSG-7701 (Fig. 1C). Additionally, based on the clinical data of 288 HCC samples in TCGA, high miR-500a expression in HCC tissues was significantly correlated with the poor prognosis of patients (Fig. 1D). Thus, we concluded that miR-500a expression is increased in HCC, and high miR500a expression predicts poor prognosis.

Increased miR-500a in HCC is mediated by deregulated histone modifications

We next examined the underlying mechanisms for the increased expression of miR500a in HCC. Deregulated histone modifications have been suggested to be important 
for the gene expression profile in cancer. For example, acetylation and tri-methylation of histone $\mathrm{H} 3$ at Lys4 (H3K4me3) are linked to transcriptional activation, while tri-methylation of $\mathrm{H} 3$ at $\mathrm{K} 9$ (H3K9me3) or K27 (H3K27me3) is linked to transcriptional repression [13]. We performed ChIP-quantitative PCR analysis to detect the methylation and acetylation of histone $\mathrm{H} 3$ in the miR-500a promoter region. The results showed that the levels of H3K4me3 in miR-500a promoter region were significantly increased in HCC SMMC-7721 and HepG2 cells as compared with the liver cell line QSG-7701, and H3K9me3 and H3K27me3 levels were significantly decreased. Moreover, the acetylation level of $\mathrm{H} 3$ was also significantly increased in HCC cells (Fig. 2A-

D). These data suggested that the deregulated histone modifications in the miR500 a promoter region may be responsible for its increased expression in HCC.

Inhibition of miR-500a
promotes apoptosis of HCC
cells
As high miR-500a expression in HCC predicts poor prognosis, the potential roles of miR-500a in HCC progression were then investigated. Transfection of miR-500a inhibitor significantly decreased miR500a expression in the HCC cell lines SMMC-7721 and HepG2 (Fig. 3A), and the proliferation of these cells was significantly suppressed by inhibition of miR-500a (Fig. 2B-C). These data indicate that miR-500a promotes HCC progression in vitro. Furthermore, we used HCC SMMC-7721 cells to build a subcutaneous xenograft model in nude mice, and then injected antagomiR-500a$3 p$ intratumorally to inhibit
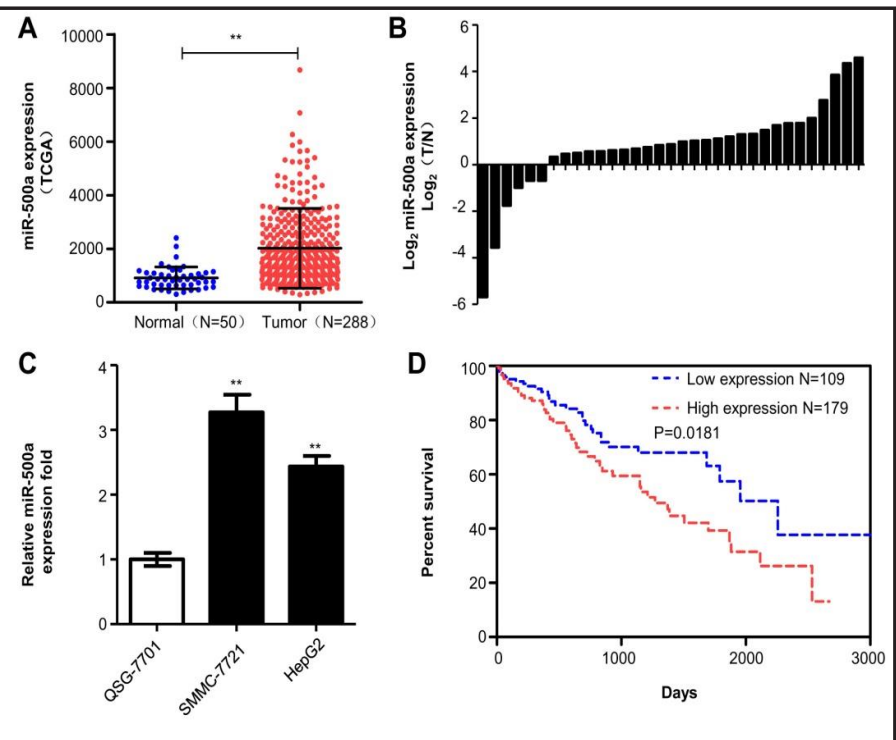

Fig. 1. miR-500a is up-regulated in HCC tissue and cell lines. A. miR-500a expression levels in 50 normal livers and 288 HCC tumor samples from TCGA RNA-Seq database. B. Quantitative real-time PCR (qRT-PCR) analysis of miR-500a expression in 33 matched human HCC and corresponding adjacent normal tissues. C. Relative expression of miR-500a in the immortalized normal hepatic cell line QSG-7701 and HCC cell lines SMMC-7721 and HepG2 was detected by qRT-PCR. The expression of miR-500a was normalized to that of U6 in each sample. D. Kaplan-Meier survival curves showing the relationship between high or low miR-500a expression levels (average value was chosen as the cutoff point) with the overall survival of HCC patients (288 clinical samples from TCGA). The results are expressed as the mean $\pm \operatorname{SD}\left({ }^{* *} \mathrm{p}<0.01\right)$. the expression if miR-500a in

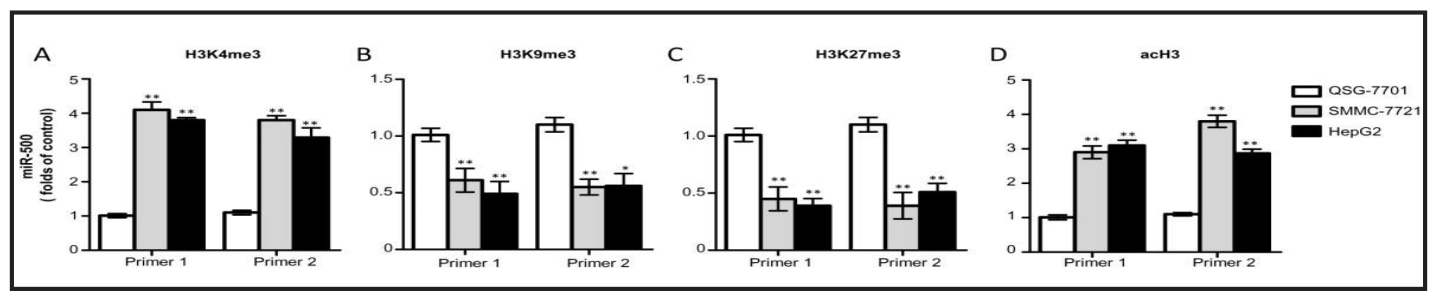

Fig. 2. Histone modifications mediate the upregulation of miR-500a expression in HCC. A-D. qRT-PCR analysis for H3K4me3, H3K9me3, H3K27me3, and acH3 regulation of miR-500a by ChIP assay in the immortalized normal hepatic cell line QSG-7701 and HCC cell lines SMMC-7721 and HepG2. The results are expressed as the mean $\pm \mathrm{SD}\left({ }^{*} \mathrm{p}<0.05,{ }^{* *} \mathrm{p}<0.01\right)$.

\section{KARGER}


vivo. As compared with the control antagomiR-NC group, transplanted tumor growth was significantly suppressed by the administration of antagomiR-500a-3p (Fig. 2D). Thus, high miR-500a expression in HCC promotes tumor growth both in vivo and in vitro. In addition, we examined the roles of miR-500a in regulating HCC apoptosis, and found that inhibition of miR-500a promoted the apoptosis of HCC cells, as determined using flow cytometry (Fig. 2E). Together, these data suggest that high miR-500a expression in HCC may inhibit HCC apoptosis to promote progression.

\section{miR-500a directly targets BID}

To investigate the underlying mechanism for the apoptosis-inhibiting role of miR-500a in HCC cells, we used the TargetScan prediction method to identify potential targets of miR-500a. Among the mRNAs predicted to be targeted by miR-500a, the Bcl-2 family member BID was the most closely related to apoptosis and contributes to the induction of apoptosis (Fig. 3A). Therefore, we constructed a dual luciferase reporter system containing the 3'-UTR region of BID and found that luciferase activity was significantly reduced by co-transfection with miR-500a mimics, whereas a luciferase reporter containing a mutated potential target site in the $3^{\prime}$-UTR of BID failed to be targeted by miR-500a (Fig. 4B). Moreover, BID protein expression was also enhanced by inhibition of miR-500a in HCC HepG2 and SMMC-7721 cells (Fig. 4C). These results indicate that BID mRNA is targeted directly by miR-500a, which may contribute to its apoptosis-inhibiting role in HCC.

It is well known that BID can induce cell apoptosis via the tBID-mitochondrial pathway [17]. Therefore, we examined the activation of the BID downstream pathway in HCC HepG2 and SMMC-7721 cells. Inhibition of miR-500a significantly increased the levels of tBID and cytochrome $\mathrm{C}$ in the mitochondria and enhanced caspase- 3 and caspase- 9 activation (Fig.
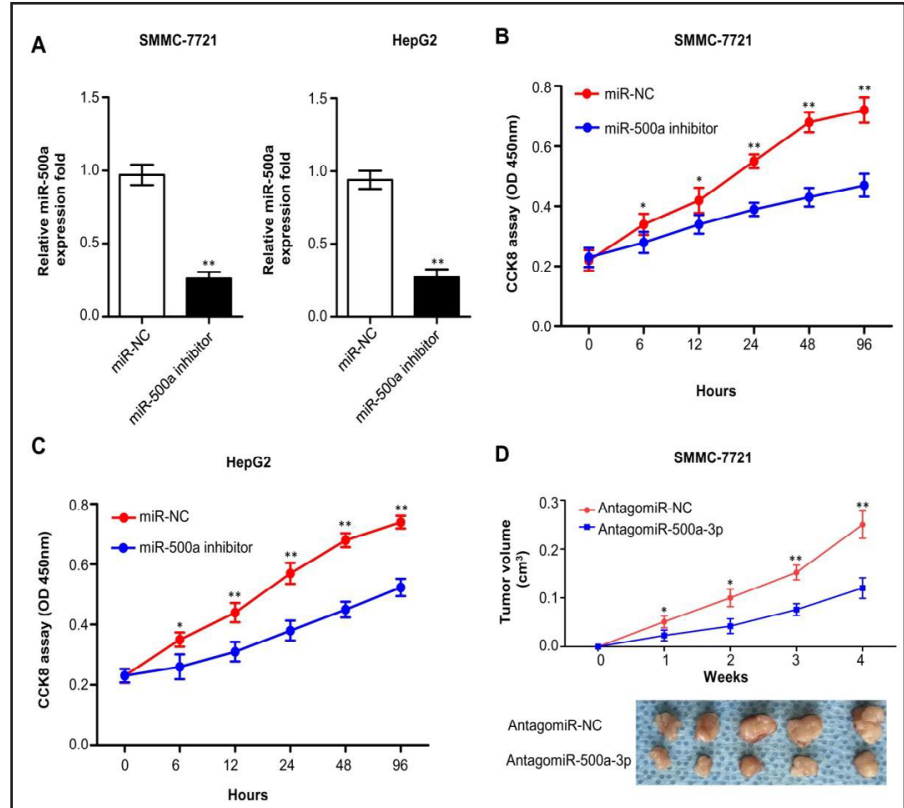

D $\quad$ sMmc-7721
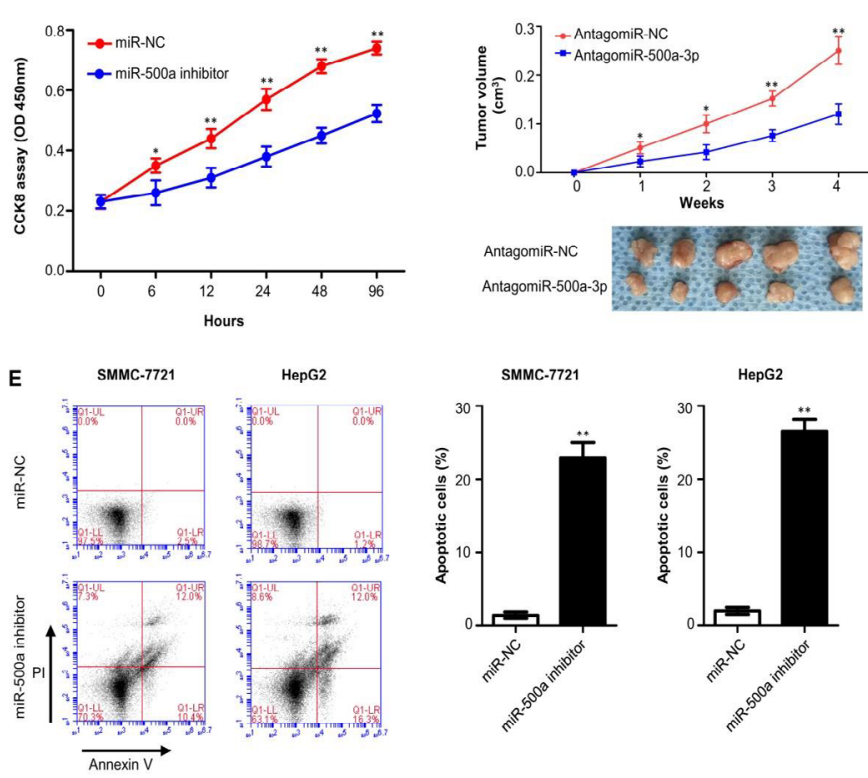

Fig. 3. Inhibition of miR-500a promotes HCC apoptosis and reduces proliferation. A. Relative expression of miR-500a in SMMC-7721 and HepG2 cells transfected with $80 \mathrm{nM}$ miR-500a inhibitor or control. B-C. Estimation of cell proliferation in SMMC-7721 and HepG2 cells transfected with control or miR-500a inhibitor by CCK-8 assays. D. Biological function of miR-500a on tumorigenicity in a nude mouse xenograft model. SMMC-7721 cells $\left(2.0 \times 10^{6}\right.$ cells $)$ were injected subcutaneously into the skin of nude mice and allowed to grow for 2 weeks. Then, antagomiR-NC or antagomiR-500a was injected intravenously into the tumors once every 3 days for 2 weeks. E. Rate of apoptosis of SMMC-7721 and HepG2 cells transfected with miR500 a inhibitor or miR-NC was detected by FACS. The results are expressed as the mean \pm SD $\left({ }^{*} \mathrm{p}<0.05,{ }^{* *} \mathrm{p}<0.01\right)$. 
Fig. 4. miR-500a directly targets BID. A. TargetScan analysis demonstrated the presence of putative miR-500a targets sites in the 3 '-UTR of BID. Schematic showing the putative binding sites of miR-500a in the $3^{\prime}$ UTR of BID and mutations in the miR-500a binding sites. B. Relative luciferase activity in BID 3'-UTR wild-type (WT) and BID 3'-UTR mutant (Mut) demonstrated that miR-500a mimics downregulate luciferase activity from wild-type BID 3 '-UTR, but not from mutant BID 3'-UTR. C. Western blot analysis of BID expression in SMMC-7721 and HepG2 cells transfected with miR-NC and miR-500a inhibitor. D. Western blot analysis of the tBID-mitochondrial pathway in SMMC-7721 and HepG2 cell lines transfected with miRNC or miR-500a inhibitor. The results are expressed as the mean \pm SD $\left({ }^{* *} p<0.01\right.$, ns means not significant).

Fig. 5. miR-500a inhibits HCC apoptosis by the post-transcriptional regulation of BID. A. qRT-PCR analysis of miR-500a expression in SMMC-7721 and HepG2 cells transfected with miR-NC or miR-500a inhibitor, followed by treatment with ABT-199 $(2 \mu \mathrm{M})$ or DMSO, as indicated. B. Rate of apoptosis of SMMC7721 and HepG2 cells transfected with miR500 a inhibitor or miR-NC and treated with ABT-199 $(2 \mu \mathrm{M})$ or DMSO was detected by FACS. The results are expressed as the mean $\pm \mathrm{SD}\left({ }^{* *} \mathrm{p}<0.01\right.$, ns means not significant).
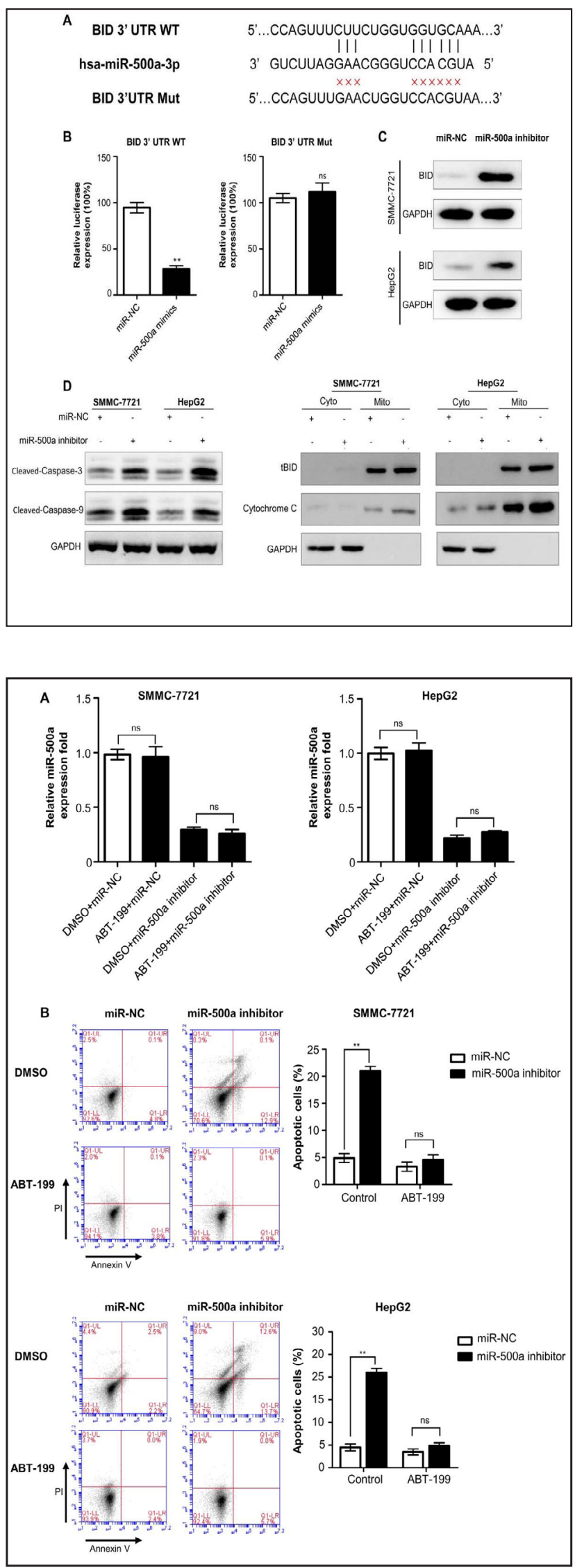
4D). These results suggest that miR-500a inhibits HCC apoptosis by targeting the tBIDmitochondrial apoptosis pathway.

\section{BID is responsible for the miR-500a-mediated inhibition of apoptosis}

To further clarify the role of BID in the miR-500a-mediated inhibition of apoptosis, we treated HCC SMMC-7721 and HepG2 cells with the Bcl-2 inhibitor ABT-199. miR-500a expression was not influenced by ABT-199 administration in SMMC-7721 and HepG2 cells (Fig. 5A), and we found that the miR-500a-mediated inhibition of apoptosis was almost abolished by ABT-199 administration (Fig. 5B). Thus, these data demonstrate that miR-500a exerts its apoptosis-inhibiting function mainly through targeting the apoptosis inducer BID.

\section{Discussion}

Previous studies have shown that a set of miRNAs was deregulated in HCC, and some of them play important roles in the carcinogenesis and progression of HCC[18-21]. In addition, some of these deregulated miRNAs can be used as diagnostic or prognostic markers. For miR-500a, it was suggested that it was increased in the sera of HCC patients and returned to normal after surgical resection; thus, it may be a diagnostic marker for human HCC [22]. It was also suggested that miR-500a may be important for the maintenance of cancer stem cell properties [23]. In the present study, we found that miR-500a was significantly increased in HCC tissues, and high miR-500a expression predicted poor prognosis, thus suggesting that miR-500a may be a potential prognostic and therapeutic target of HCC.

The biosynthesis and degradation of miRNAs are regulated elaborately, and their regulation includes epigenetic, transcription factor, and post-transcriptional processing mechanisms. Among them, the epigenetic regulation of miRNA expression is of great importance, especially histone modifications. Histone modifications include methylation, acetylation, and other modification processes. The trimethylation of histone is stable, and trimethylation at different loci can generate different effects. Generally, the methylation of $\mathrm{H} 3 \mathrm{~K} 4, \mathrm{H} 3 \mathrm{~K} 36$, and H3K79 is related to transcriptional activation, while the methylation of H3K9, H3K27, and H4K20 exerts an inhibitory effect on transcription. Meanwhile, histone acetylation can enhance the transcriptional level of a gene temporarily [24]. Similarly, our data show that the upregulation of miR-500a in HCC cells may be mediated by the upregulation of histone H3K4 methylation, downregulation of H3K9 and H3K27 methylation, and upregulation of $\mathrm{H} 3$ acetylation.

BID protein, which is related to the death receptor pathway and mitochondrial pathwayinduced apoptosis, is very effective at promoting apoptosis [25]. BID is mainly located in the cytoplasm and does not show pro-apoptotic activity. However, when caspase-8 is activated, BID is recognized and cleaved to the activated form of the protein, termed as tBID, and tBID is transported from the cytoplasm to the outer mitochondrial membrane to combine with Bcl2 protein and inhibit its anti-apoptotic effect $[17,26]$ or increase mitochondrial permeability to induce the release of cytochrome $\mathrm{C}$, thus activating downstream caspase- 3 and caspase- 9 to promote apoptosis [27]. Recently, miR-20a was found to target the BID gene directly and induce apoptosis in the TRAIL-resistant rectal cancer cell line SW480 through the tBIDmitochondrial pathway. tBID enhances apoptosis via the mitochondrial pathway [28] only in specific types of cell, namely, CD95 type I cells [29]. Some studies have shown that apoptosis in HepG2 and SMMC-7721 [30, 31] cells was regulated by the tBID-mediated-mitochondrial pathway, which is in accordance with the findings of the present study. The discovery that miR-500a inhibits cell apoptosis by suppressing the tBID-mitochondrial apoptosis pathway adds a new aspect to the regulation of apoptosis by BID.

An miRNA often has a set of targets and then regulates multiple signaling pathways involved in cancer development [32]. For miR-122, it can inhibit proliferation and induce apoptosis by directly targeting cyclin G1 [33] or by directly targeting the Wnt/ $\beta$-catenin pathway [34], and can even affect the metabolism of HCC by directly targeting pyruvate 
kinase M2 [35]. Recently, miR-500a was reported to directly target SFRP2 and GSK-3 $\beta$ mRNA and promote cancer migration and invasion [36]. However, in the present study, a series of in vivo and in vitro experiments demonstrated that miR-500a can promote the development of HCC by targeting BID and inhibiting the tBID-mitochondrial apoptosis pathway. In addition, investigating the entire targetome of miR-500a in future studies may identify additional roles for this miRNA in HCC.

\section{Acknowledgements}

This study was supported by the National Natural Science Foundation of China (Nos. 81373171, 31370896, 81471585, and 31470873).

\section{Disclosure Statement}

The authors declare that they have no actual or potential conflicts of interest.

\section{References}

1 Wang ZG, Zheng H, Gao W, Han J, Cao JZ, Yang Y, Li S, Gao R, Liu H, Pan ZY, Fu SY, Gu FM, Xing H, Ni JS, Yan HL, Ren H, Zhou WP: eIF5B increases ASAP1 expression to promote HCC proliferation and invasion. Oncotarget 2016;7:62327-62339.

-2 Cancer Genome Atlas Research Network. Electronic address wbe, Cancer Genome Atlas Research $\mathrm{N}$ : Comprehensive and Integrative Genomic Characterization of Hepatocellular Carcinoma. Cell 2017;169:1327-1341 e1323.

-3 Sintra SN, Tome L, Cipriano MA, Bento C, Furtado E: Long-term outcome of the first 150 liver transplant recipients: a single-center experience. Transplant Proc 2013;45:1119-1121.

4 Gal-Yam EN, Saito Y, Egger G, Jones PA: Cancer epigenetics: modifications, screening, and therapy. Annu Rev Med 2008;59:267-280.

5 Pan Y, Li C, Chen J, Zhang K, Chu X, Wang R, Chen L: The Emerging Roles of Long Noncoding RNA ROR (lincRNA-ROR) and its Possible Mechanisms in Human Cancers. Cell Physiol Biochem 2016;40:219-229.

-6 Sui J, Yang X, Qi W, Guo K, Gao Z, Wang L, Sun D: Long Non-Coding RNA Linc-USP16 Functions As a Tumour Suppressor in Hepatocellular Carcinoma by Regulating PTEN Expression. Cell Physiol Biochem 2017;44:1188-1198.

7 Du Y, Liu XH, Zhu HC, Wang L, Ning JZ, Xiao CC: MiR-543 Promotes Proliferation and EpithelialMesenchymal Transition in Prostate Cancer via Targeting RKIP. Cell Physiol Biochem 2017;41:1135-1146.

-8 Sun JJ, Chen GY, Xie ZT: MicroRNA-361-5p Inhibits Cancer Cell Growth by Targeting CXCR6 in Hepatocellular Carcinoma. Cell Physiol Biochem 2016;38:777-785.

-9 Saito Y, Hibino S, Saito H: Alterations of epigenetics and microRNA in hepatocellular carcinoma. Hepatol Res 2014;44:31-42.

10 Krol J, Loedige I, Filipowicz W: The widespread regulation of microRNA biogenesis, function and decay. Nat Rev Genet 2010;11:597-610.

11 Rupaimoole R, Calin GA, Lopez-Berestein G, Sood AK: miRNA Deregulation in Cancer Cells and the Tumor Microenvironment. Cancer Discov 2016;6:235-246.

12 Beermann J, Piccoli MT, Viereck J, Thum T: Non-coding RNAs in Development and Disease: Background, Mechanisms, and Therapeutic Approaches. Physiol Rev 2016;96:1297-1325.

13 Hou J, Lin L, Zhou W, Wang Z, Ding G, Dong Q, Qin L, Wu X, Zheng Y, Yang Y, Tian W, Zhang Q, Wang C, Zhang Q, Zhuang SM, Zheng L, Liang A, Tao W, Cao X: Identification of miRNomes in human liver and hepatocellular carcinoma reveals miR-199a/b-3p as therapeutic target for hepatocellular carcinoma. Cancer Cell 2011;19:232-243. 


\section{Cellular Physiology Cell Physiol Biochem 2018;47:2046-2055 \begin{tabular}{l|l} 
and Biochemistry & $\begin{array}{l}\text { DOI: 10.1159/000491472 } \\
\text { Published } 2018 \text { The Author(s). Published by S. Karger AG, Basel }\end{array}$ \\
\hline
\end{tabular} \\ Published online: July 03,2018}

14 Zhan Y, Zheng N, Teng F, Bao L, Liu F, Zhang M, Guo M, Guo W, Ding G, Wang Q: MiR-199a/b-5p inhibits hepatocellular carcinoma progression by post-transcriptionally suppressing ROCK1. Oncotarget 2017;8:67169-67180.

15 Zhang L, Ding Y, Yuan Z, Liu J, Sun J, Lei F, Wu S, Li S, Zhang D: MicroRNA-500 sustains nuclear factorkappaB activation and induces gastric cancer cell proliferation and resistance to apoptosis. Oncotarget 2015;6:2483-2495.

16 Cai B, Chen W, Pan Y, Chen H, Zhang Y, Weng Z, Li Y: Inhibition of microRNA-500 has anti-cancer effect through its conditional downstream target of TFPI in human prostate cancer. Prostate 2017;77:1057-1065.

17 Li H, Zhu H, Xu CJ, Yuan J: Cleavage of BID by caspase 8 mediates the mitochondrial damage in the Fas pathway of apoptosis. Cell 1998;94:491-501.

18 Wahid B, Ali A, Rafique S, Idrees M: New Insights into the Epigenetics of Hepatocellular Carcinoma. Biomed Res Int 2017;2017:1609575.

19 Bie B, Sun J, Li J, Guo Y, Jiang W, Huang C, Yang J, Li Z: Baicalein, a Natural Anti-Cancer Compound, Alters MicroRNA Expression Profiles in Bel-7402 Human Hepatocellular Carcinoma Cells. Cell Physiol Biochem 2017;41:1519-1531.

20 Liu Y, Chai Y, Zhang J, Tang J: A Function Variant at miR-501 Alters Susceptibility to Hepatocellular Carcinoma in a Chinese Han Population. Cell Physiol Biochem 2016;38:2500-2508.

-21 Yang Y, Liu Y, Xue J, Yang Z, Shi Y, Shi Y, Lou G, Wu S, Qi J, Liu W, Chen Z, Wang J: MicroRNA-141 Targets Sirt1 and Inhibits Autophagy to Reduce HBV Replication. Cell Physiol Biochem 2017;41:310-322.

22 Yamamoto Y, Kosaka N, Tanaka M, Koizumi F, Kanai Y, Mizutani T, Murakami Y, Kuroda M, Miyajima A, Kato T, Ochiya T: MicroRNA-500 as a potential diagnostic marker for hepatocellular carcinoma. Biomarkers 2009;14:529-538.

-23 Jiang C, Long J, Liu B, Xu M, Wang W, Xie X, Wang X, Kuang M: miR-500a-3p promotes cancer stem cells properties via STAT3 pathway in human hepatocellular carcinoma. J Exp Clin Cancer Res 2017;36:99.

-24 Lachner M, O’Sullivan RJ, Jenuwein T: An epigenetic road map for histone lysine methylation. J Cell Sci 2003;116:2117-2124.

25 Billen LP, Shamas-Din A, Andrews DW: Bid: a Bax-like BH3 protein. Oncogene 2008;27 Suppl 1:S93-104.

26 Orzechowska EJ, Girstun A, Staron K, Trzcinska-Danielewicz J: Synergy of BID with doxorubicin in the killing of cancer cells. Oncol Rep 2015;33:2143-2150.

27 Billard C: Design of novel BH3 mimetics for the treatment of chronic lymphocytic leukemia. Leukemia 2012;26:2032-2038.

28 Huang G, Chen X, Cai Y, Wang X, Xing C: miR-20a-directed regulation of BID is associated with the TRAIL sensitivity in colorectal cancer. Oncol Rep 2017;37:571-578.

29 Barnhart BC, Alappat EC, Peter ME: The CD95 type I/type II model. Semin Immunol 2003;15:185-193.

30 Zhou H, Xu M, Gao Y, Deng Z, Cao H, Zhang W, Wang Q, Zhang B, Song G, Zhan Y, Hu T: Matrine induces caspase-independent program cell death in hepatocellular carcinoma through bid-mediated nuclear translocation of apoptosis inducing factor. Mol Cancer 2014;13:59.

-31 Zhang P, Huang CR, Wang W, Zhang XK, Chen JJ, Wang JJ, Lin C, Jiang JW: Harmine Hydrochloride Triggers G2 Phase Arrest and Apoptosis in MGC-803 Cells and SMMC-7721 Cells by Upregulating p21, Activating Caspase-8/Bid, and Downregulating ERK/Bad Pathway. Phytother Res 2016;30:31-40.

-32 Lewis BP, Burge CB, Bartel DP: Conserved seed pairing, often flanked by adenosines, indicates that thousands of human genes are microRNA targets. Cell 2005;120:15-20.

-33 Gramantieri L, Ferracin M, Fornari F, Veronese A, Sabbioni S, Liu CG, Calin GA, Giovannini C, Ferrazzi E, Grazi GL, Croce CM, Bolondi L, Negrini M: Cyclin G1 is a target of miR-122a, a microRNA frequently downregulated in human hepatocellular carcinoma. Cancer Res 2007;67:6092-6099.

-34 Xu J, Zhu X, Wu L, Yang R, Yang Z, Wang Q, Wu F: MicroRNA-122 suppresses cell proliferation and induces cell apoptosis in hepatocellular carcinoma by directly targeting Wnt/beta-catenin pathway. Liver Int 2012;32:752-760.

-35 Liu AM, Xu Z, Shek FH, Wong KF, Lee NP, Poon RT, Chen J, Luk JM: miR-122 targets pyruvate kinase M2 and affects metabolism of hepatocellular carcinoma. PLoS One 2014;9:e86872.

-36 Guo Y, Chen L, Sun C, Yu C: MicroRNA-500a promotes migration and invasion in hepatocellular carcinoma by activating the Wnt/beta-catenin signaling pathway. Biomed Pharmacother 2017;91:13-20. 